\title{
The MoEDAL experiment - a new light on LHC physics
}

\author{
James L. Pinfold ${ }^{1, a}$ \\ ${ }^{1}$ Physics Department \\ University of Alberta \\ Edmonton, Alberta T6G 0V1 \\ Canada.
}

\begin{abstract}
In 2010 the MoEDAL experiment at the Large Hadron Collider (LHC) was unanimously approved by CERN's Research Board to start data taking in 2015. MoEDAL is a pioneering experiment designed to search for highly ionizing messengers of new physics such as magnetic monopoles or massive (pseudo-)stable charged particles. Its groundbreaking physics program defines a number of scenarios that yield potentially revolutionary insights into foundational questions. MoEDAL's purpose is to meet such farreaching challenges at the frontier of the field. The innovative MoEDAL detector is tuned to the prospect of discovery physics. The largely passive MoEDAL detector, deployed at Point 8 on the LHC ring, has a dual nature. First, it acts like a giant camera, comprised of nuclear track detectors - analyzed offline by ultra fast scanning microscopes - sensitive only to new physics. Second, it is uniquely able to trap the particle harbingers of new physics beyond the Standard Model for further study. MoEDAL's radiation environment is monitored by a state-of-the-art real-time TimePix pixel detector array.
\end{abstract}

\section{Introduction}

In 2010, the CERN Research Board (RB) unanimously approved MoEDAL [1] to run in 2015 and take data at an energy of $14 \mathrm{TeV}$. The LHC provides an extraordinary new light at the high-energy frontier allowing direct exploration of the fundamental physical laws governing the evolution of the Universe just a fraction of a second after the Big Bang when particle energies were in the $\mathrm{TeV}$ range - the Terascale. The MoEDAL experiment is a groundbreaking experiment designed to search for Highly Ionizing Particle (HIP) avatars of new physics at the Terascale. The"high-risk" nature of MoEDAL's extensive physics program is compelling not only because of the prospect of a revolutionary breakthrough with impact far beyond the realm of particle physics, but also by the certitude of unique and extensive search constraints it can place on new physics.

The MoEDAL detector shares Intersection Point 8 (IP8) on the LHC ring with LHCb as shown in Figure 1. It utilizes an innovative combination of passive and active detection techniques employed by particle and astroparticle experiments. The MoEDAL experiment has a huge discovery potential designed to expand the physics horizons of the LHC in a complementary way. Its groundbreaking physics program, presented in our recent paper [2], defines a number of scenarios each of which could revolutionize our fundamental understanding of nature providing insights into such foundational

a e-mail: jpinfold@ualberta.ca 
questions as: are there extra dimensions or new symmetries of nature; what is the mechanism for the generation of mass; does magnetic charge exist; what is the nature of dark matter; and, how did the Universe develop at the earliest times.
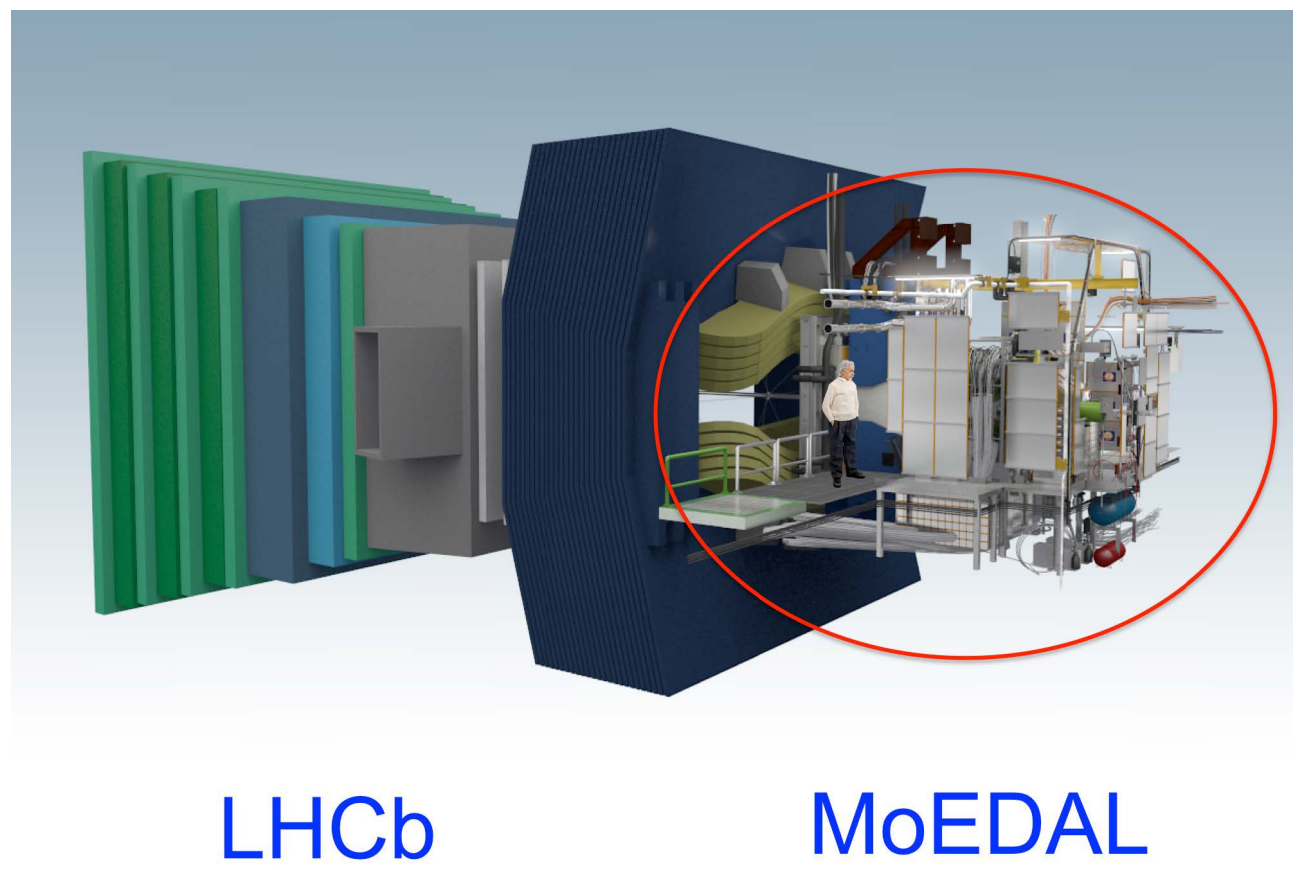

Figure 1. A schematic view of the MoEDAL detector and its deployment at IP8

The unprecedented but complementary nature of the MoEDAL detector and its systematics - exemplified by its ability to retain a permanent record, and even capture new particles for further study - will make MoEDAL an invaluable asset in the elucidation of any Terascale Beyond the Standard Model (BSM) scenario covered by its extensive physics repertoire.

\section{The MoEDAL Detector}

The novel MoEDAL detector, deployed at IP8 on the LHC ring, is comprised of four subsystems as shown in Figure 2. The main subsystem, referred to as the Low Threshold Nuclear Track Detector (LT-NTD) array, is the largest array of (400) plastic NTD stacks $\left(25 \times 25 \mathrm{~cm}^{2}\right.$ ever deployed at an accelerator, comprised of CR39 (4), MAKROFOL (3) and lexan (3) plastic NTD sheets. The CR39 NTDs have an excellent charge resolution ( 0.2 of a single electric charge, based on a single measurement) and low threshold, allowing the detection of HIPs with and ionizing power equal to or more than 5 times that of a Minimum Ionizing Particle (MIP). The Nuclear Track detectors (NTDs) are first etched and subsequent scanned in order to reveal the ionization trail of the magnetic monopole as shown in Figure 3. The velocity dependent ionization energy loss signature of a magnetic monopole is characteristic and different from an electrically charged particle. 
The LT-NTD array has been enhanced by the High Charge Catcher (HCC) sub-detector - with threshold around 50 MIPS - comprised of flexible low mass stacks with 3 MAKROFOL sheets in an Al-foil envelope applied directly in areas adjacent to, or on, LHCB's VELO structures otherwise unsuitable for LT-NTD detectors - increasing the overall geometrical acceptance for monopoles of the NTD system to $60 \%$. The exposed NTD detectors will be analyzed at Bologna and CERN using state-of-the art ultra-fast automated optical scanning microscopes developed by the Muenster and INFN-Bologna MoEDAL groups, combined with cutting edge image analysis software. This enables low NTD thresholds to be maintained despite large beam related backgrounds. The NTD detectors will be calibrated using the heavy-ion (Fe) beam at NASA's Space Radiation Laboratory (NSRL)

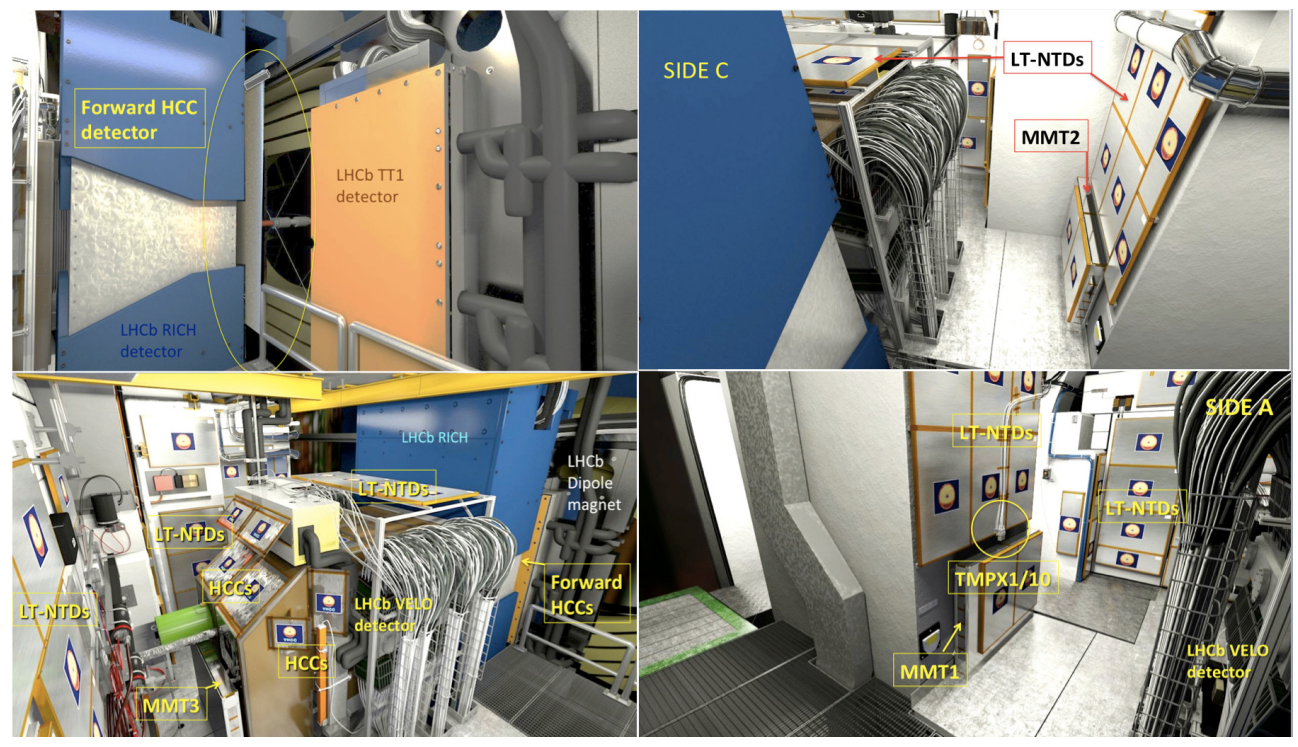

Figure 2. Views of a visualization of the MoEDAL detector showing elements of all 4 sub-detector systems

The Magnetic Monopole Trapper (MMT) is the third MoEDAL sub-detector system. This detector system relies on a SQUID, essentially a superconducting coil of wire, to detect magnetic charge trapped in the MMT detector volume. If a magnetic monopole passes through the ring, the changing magnetic field induces a stable current, which can be measured. The current jump when the monopole traverses the SQUID coil gives an accurate measure of the strength of the magnetic charge. This method of detection, illustrated in Figure 4, is independent of the monopole mass or velocity.

The sensitive volume of the MMT consists of roughly 1 tonne of pure aluminium (Al) trapping volumes deployed around the MoEDAL cavern at IP8. Al is well suited as it has an anomalously large nuclear magnetic moment. A fraction of the massive HIPs created will stop and be captured in the MMT detector. After exposure the MMT trapping volumes will be monitored at the ETH SQUID facility for the presence captured monopoles. Test solenoids can be used to calibrate the response of the SQUID to a trapped monopole. After the SQUID scan has been performed the trapping volumes will be sent to an underground laboratory, for example SNOLAB, to be monitored for the decays of very long lived electrically charged particles.

The fourth and only active sub-detector system is the TimePix pixel device [3] array (TMPX), consisting of $\sim 8$ devices distributed throughout the MoEDAL cavern at IP8. TMPX will be used 

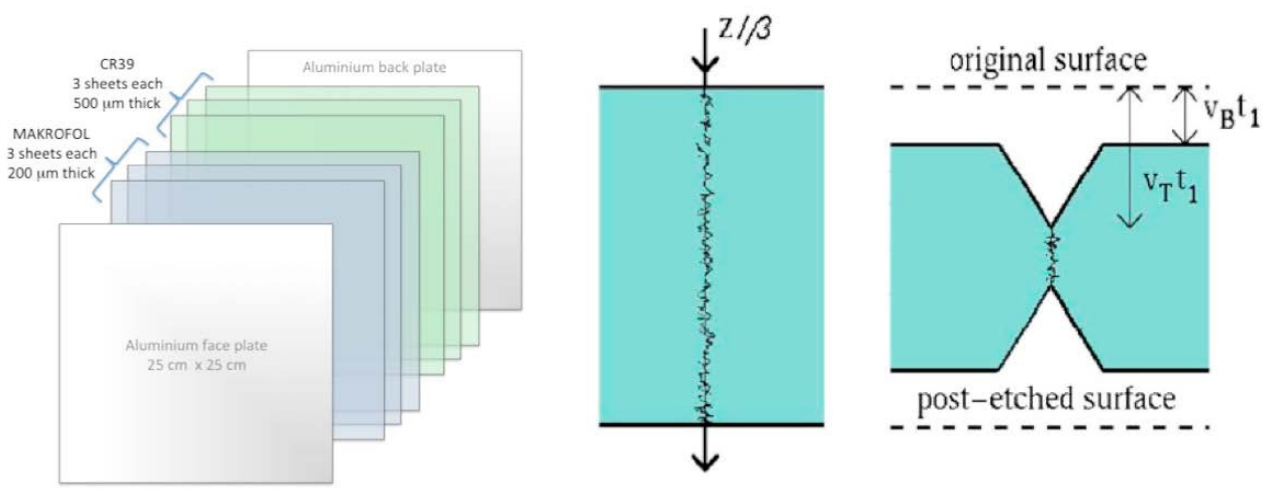

Figure 3. (Left) A MoEDAL stack comprised of CR39 and Makrofol NTD detectors. (Right) the damage zone created by an HIP that is subsequently revealed by etching in a hot $\mathrm{HaOH}$ solution

to monitor highly ionizing beam related backgrounds. Each pixel of the innovative TimePix chip contains a preamp, a discriminator with threshold adjustment, synchronization logic and a 14-bit counter. MoEDAL uses TimePix Time-over- Threshold modes where each pixel acts as an ADC for energy measurement. Effectively, the TimePix device is a tiny electronic bubble-chamber providing a real-time "colour" (energy dependent) movie of the background.
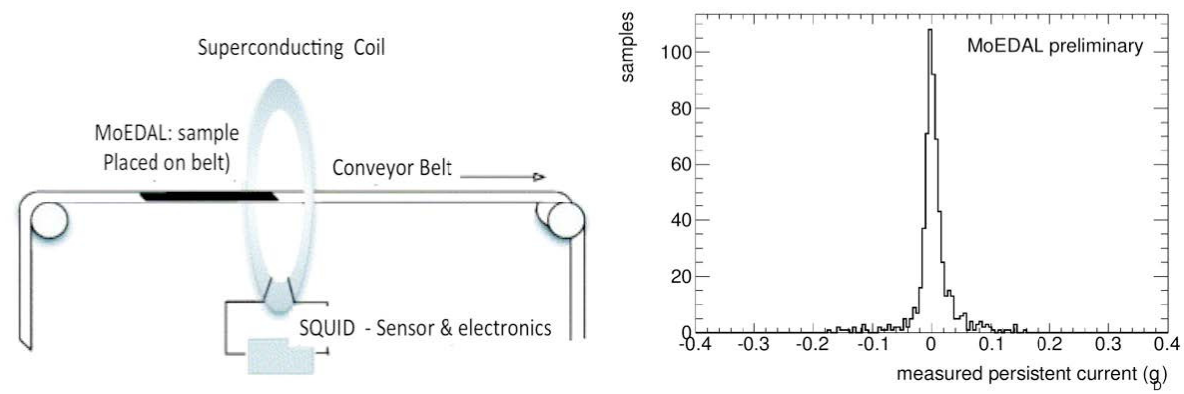

Figure 4. (Left) A schematic depiction of the use of a SQUID magnetometer to detect the presence of a trapped magnetic charge. (Right) The results of a SQUID magnetometer scan of MMT elements exposed to collisions as part of a MoEDAL test detector.

The MoEDAL NTD and MMT arrays are completely passive requiring no readout electronics, high voltage, gas supply or trigger. The NTD plastic will be replaced twice for each period of p-p running. The Al MMT volumes will be replaced only once per year. The exposed NTD plastic and Al MMT volumes will be sent to remote sites for analysis. A separate set of NTD plastic stacks will be utilized for the short heavy-ion run that takes place after the period of $p$-p running. Thus, MoEDAL has two novel ways to register the presence of new physics. It is envisaged that MoEDAL will run for five yearly periods requiring the various detection volumes to be changed at least five times, in order to reach MoEDAL's approved integrated luminosity. As the TimePix pixel device array is accessed via the web, it is not necessary to continuously man MoEDAL. 

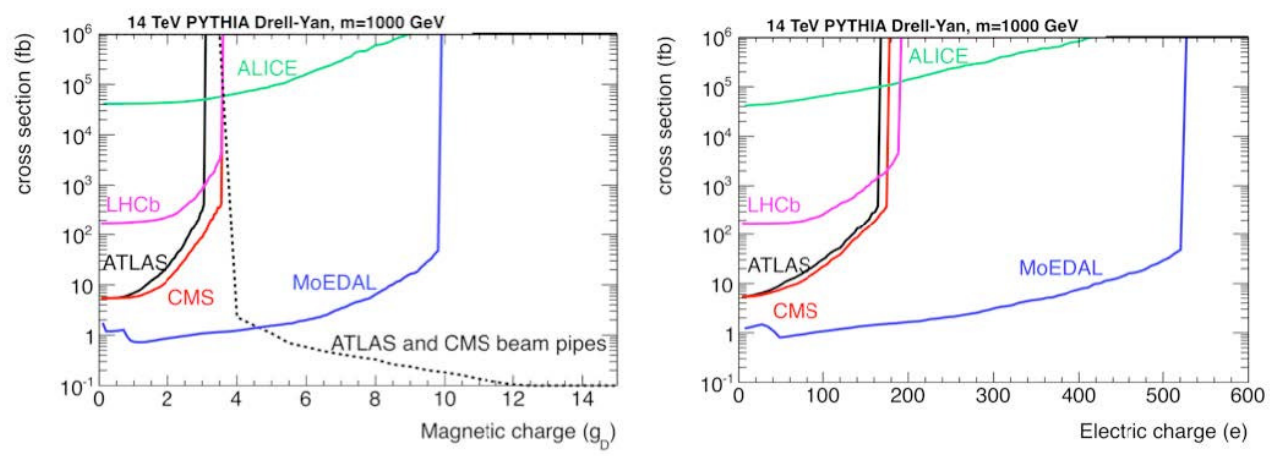

Figure 5. The expected reach [4] of the search for direct monopole anti-monopole Drell-Yan pair production process at the LHC $(\mathrm{Ecm}=14 \mathrm{TeV})$. Assuming the luminosity taken by LHCb/MoEDAL is $2 \mathrm{fb}^{-1}$, by ATLAS \& CMS $20 \mathrm{fb}^{-1}$, and by ALICE $0.004 \mathrm{fb}^{-1}$.

Any HIP BSM particle crossing an NTD stack within 450 angle to the normal will be detected with $100 \%$ efficiency. The signal for a HIP is a set of 20 equal sized etch pits - aligned with a precision of $10 \mu \mathrm{ms} /$ pit pointing towards IP8. In the case of a monopole nearing the end of its range the etch pits will be decreasing in size as the monopole ionizing power decreases with speed, unlike electrically charged particles. Although, the low energy highly ionizing backgrounds can "fog the film" they cannot "fake" a signal. There is no Standard Model (SM) background to HIP messengers of new physics in the NTD or MMT detectors.

Standard collider detectors are not optimized for the detection of HIPs such as magnetic monopoles and massive long-lived or stable charged particles. Rather, the general-purpose detectors, such as ATLAS and CMS, are designed to detect minimum-ionizing particles moving near to the speed of light. Effects arising from the particles low velocity and high-density energy deposition, such as electronics saturation, light quenching in scintillators, and adjacent hits from delta electrons, are extremely challenging to deal with. Indeed, in some cases it may be impossible to make an accurate measurement of the effective charge of the particle. For example, the resulting dead time as a result of electronics saturation may be of the order of the bunch crossing time. In addition HIPS will be quickly absorbed within the detector mass even before they penetrate far into the inner tracking detectors.

On the other hand, MoEDAL does not suffer from the problems that would severely hamper detection of slow moving HIPS at the main LHC detectors. The NTD based sub-detectors have the demonstrated ability to measure accurately the ionizing power of a HIP. The MMT sub-detector, read out through a remote SQUID, can directly detect magnetic charge - no other Collider detector has this capability. MoEDAL's largely passive detection systems require no trigger, electronics, high voltage or gas supply. Its light and uniform detection technology requires minimal support structure and provides maximum cost effective coverage close to IP8 with few experimental compromises.

The main LHC detectors typically require a largish statistical sample ( $\sim 100$ events $)$ to establish a signal in the presence of SM backgrounds. MoEDAL has no trigger or electronic readout, thus, slowly moving HIPs $(\beta<0.5)$ particles are no problem and only a few candidate events are enough to establish a signal with no SM backgrounds to fake a BSM signal. Figure 5 [4] compares of the sensitivities of the LHC detectors to HIPs. Table 1 [4] provides a qualitative summary of the various pros and cons of the various LHC experiments as regards their ability to detect magnetic monopoles. 
Table 1. Qualitative summary of the advantages/disadvantages of the LHC experiments as detectors of very highly ionizing particles [4]

\begin{tabular}{lccccc}
\hline Detector & Energy threshold & Angular cover & Luminosity & Timing required & Known efficiency \\
\hline ATLAS & medium & central & high & yes & no \\
CMS & relatively low & central & high & yes & no \\
ALICE & very low & very central & low & no & no \\
LHCb & medium & forward & medium & yes & no \\
MoEDAL & low & flul & medium & no & no \\
\hline
\end{tabular}

\subsection{Testing the MoEDAL Detector}

We are currently installing the basic MoEDAL detector infrastructure at IP8 for the first year of MoEDAL running starting in Spring 2015. This installation will be completed by Nov. 2014, before the IP8 underground area is closed to prepare for first beams in the upgraded LHC ring. Extensive successful tests and simulations of the MoEDAL detector systems have confirmed the scientific feasibility of the experiment:

- A small test array of MoEDAL plastic detectors was deployed at IP8 for LHC running at $900 \mathrm{GeV}$ in November 2009. The plastic was removed in the 2010/11winter shutdown, exposed to a Feion beam at the NSRL and then analyzed at INFN/Bologna. A paper reporting these studies is in preparation;

- In January 2011, a test array of $80 \mathrm{~m} 2$ of NTDs was deployed at IP8 to take data at $7 \mathrm{TeV}$. This plastic was removed in 2012, calibrated and analyzed. A paper reporting these studies is under preparation;

- In February 2012 we installed as a test two TimePix pixel chips of a total of 8-10 TimePix pixel chips deployed around the MoEDAL cavern. The real-time data provided by these chips is flowing and is being analyzed. A paper reporting the results is under preparation;

- In summer 2012 trapping detectors made using various Al alloys were tested at the ETH Zurich SQUID Facility. A test deployment of $176 \mathrm{~kg}$ of Al trapping detectors at IP8 was made in the same year and the results of this test were reported on the archive [5]. A paper reporting the results is in preparation;

- In 2013 and 2014, using plastic exposed for the full period, exposed to a heavy-ion beam at NSRL and then etched, we successfully tested the ability of Muenster's prototype computerized high-rate scanning microscopes to clearly reveal the signal in the presence of low energy HIP beam related backgrounds.

The NTD technology utilized by MoEDAL has been well tested in numerous heavy-ion beam tests. In fact, of the 31 accelerator-based direct searches for magnetic monopoles, 14 of which have been carried out using plastic NTDs [6]. However, prior to MoEDAL, plastic NTDs have never been utilized to perform extensive systematic searches for singly and multiply electrically charged colliderstable particles.

The use of SQUIDS to detect trapped magnetic charge has also been thoroughly tested in a number of particle and astroparticle experiments [7]. Until now these experiments have been performed on "found objects". MoEDAL is the first experiment to use purpose-made trapping volumes to capture magnetic charge. Also, MoEDAL is the first experiment to deploy trapping detectors - that will be monitored at a remote underground site (e.g. SNOLAB) - to search for long-lived massive electrically charged particles. 


\section{The Physics of MoEDAL}

MoEDAL is a pioneering experiment designed to exploit the use of Highly Ionizing Particles (HIPs) - such as magnetic monopoles or massive (pseudo-)stable charged particles - as wide ranging avatars of new physics. MoEDAL's pioneering physics prospectus [2] covers of 34 fundamentally important Beyond the Standard Model scenarios involving massive electrically and magnetically charged particles. One of the main objectives for MoEDAL is the state-of-the-art search for the magnetic charge carried by the monopole, just as the search for the Higgs was the prime motivation for the LHC and its general-purpose experiments.

\subsection{The Question of Magnetic Monopoles}

Dirac first hypothesized the existence, within the framework of Quantum Mechanics, in 1931 [8]. He envisaged that the magnetic monopole was the end of an infinitely thin infinitely long solenoid called a Dirac string. This string confined a magnetic flux that entered infinitely far away and exited through the monopole. But wouldn't this Dirac String be observable? The solution to this problem is that the string does not pose a problem as long as it is cannot be detected. The Aharonov-Bohm effect tells us that a charge particle - say an electron - picks up a phase moving round a region where a magnetic flux is confined - like the solenoid in the Aharonov-Bohm experiment. In this experiment the presence of a magnetic field confined to a solenoid introduces an extra term depending on the value of the vector potential A outside the solenoid:

$$
U=\exp \left(i q \oint_{C} \mathbf{A} \cdot d x\right)
$$

But, the Dirac string is a solenoid which is not detectable if the phase picked up by the wave function of the electron as it travels completely around the string (round contour $\mathrm{C}$ ) is equal to one. An evaluation of the above integral in the field of a monopole shows that for the string not to be detected:

$$
e^{i q g}=1 \Longrightarrow q g=2 \pi n(n=1,2,3 \ldots)
$$

In 1974 Gerard 't Hooft [9] and Alexander Polyakov [10] considered a slightly different variant of the Glashow Weinberg Salam electroweak theory that now forms the basis of the Standard Model. They instead considered an earlier model that had been proposed by Georgi and Glashow [11]. Remarkably, 't Hooft and Polyakov found that this theory would necessarily contain both electric and magnetic charges. The field configuration corresponding to this magnetic monopole is an example of a topological defect or a topological soliton. A topological soliton is a solution that cannot be continuously deformed into the vacuum solution due to some topological constraint.

The constraint we put the solutions is that their total energy is finite. The mass of these monopoles - roughly $100 \mathrm{GeV}$, determined by the energy scale associated with weak nuclear forces - was too low to be compatible with experimental results. But ' $t$ Hooft and Polyakov noticed that the structure of the SU(5) Grand Unified Theory (GUT) was similar to that of the Georgi-Glashow model and consequently it also predicted monopoles, but with the much higher mass of $10^{16} \mathrm{GeV}$. These monopoles are also able to catalyse nucleon decay [12]. The existence of magnetic monopoles is an unavoidable consequence of any theory that would describe all strong and electroweak forces by one GUT interaction [13].

The discovery of a Higgs-like boson in July 2012 by the ATLAS and CMS experiments at the Large Hadron Collider (LHC) at CERN has lead unambiguously to the completion of the puzzle of the 
Standard Model spectrum, thereby putting the electroweak theory of Glashow, Salam and Weinberg at the top of the "pantheo" of the successful physical theories that describe the microcosm. Yet, there are missing parts to this theory. For example, and explanation for charge quantization. The experimental verification of a monopole-like solution within the framework of the Standard Model would explain from first principles the quantization of the electric charge, something which the present Standard Model cannot do.

It has been asserted that the Weinberg-Salam model has no topological monopole of physical interest [10]. But Cho and Maison have pointed out that the Weinberg-Salam model does have exactly the same nontrivial second homotopy as the Georgi-Glashow model which allows the 't HooftPolyakov monopole [14]. The Cho-Maison monopole is the electroweak (EW) generalization of the Dirac's monopole, so that it could be viewed as a hybrid of Dirac and 't Hooft-Polyakov monopoles. But unlike the Dirac's monopole, it carries the magnetic charge $(4 \pi) / \mathrm{e}$. This is because in the standard model the $U(1)$ em has the period of $4 \pi$, not $2 \pi$, as it comes from the U(1) subgroup of SU(2). Thus the magnetic charge of the EW monopole is twice that of the Dirac Monopole. Recent estimates of the EW monopole [15] indicate that its mass is low enough to be detected at the LHC.

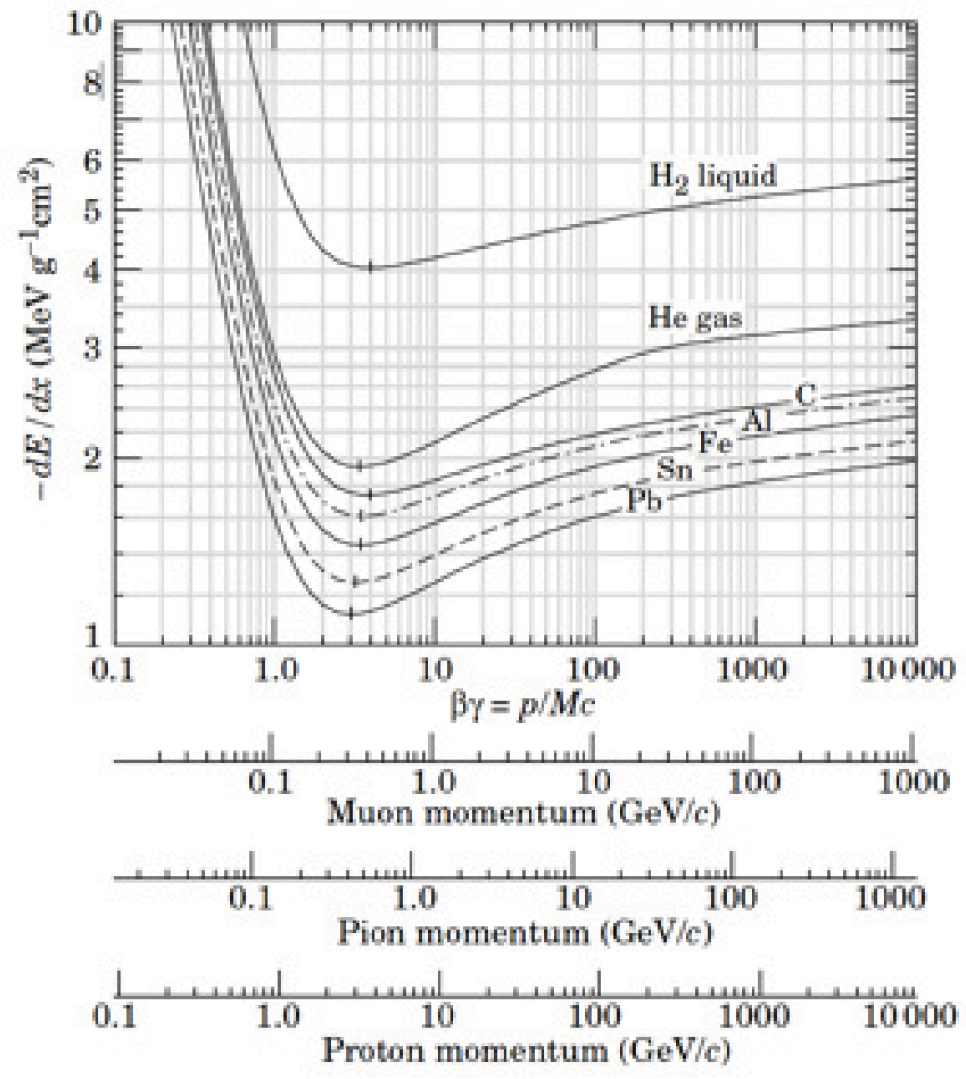

Figure 6. The energy loss for various particles in various materials as calculated using the Bethe formula 


\subsection{Highly lonising Avatars of New Physics}

To be detected a particle must interact with the material of the detector losing energy as they travel. Except when they are moving very near to the speed of light charged particles heavier than electrons primarily lose energy by interacting with the atomic electrons of the material by exciting atomic electrons or ionizing atoms. The Bethe formula [16] describes the energy loss per distance travelled of fast charged particles that are more massive than electrons such as muons, protons, pions and ions (atomic nuclei):

$$
-\frac{d E}{d x}=z^{2} \frac{K Z}{A} \frac{1}{\beta^{2}}\left[\frac{1}{2} \ln \left(\frac{2 m_{e} \beta^{2} \gamma^{2}}{I^{2}}\right)-\beta^{2}\right]
$$

where $Z e$ is the electoric charge of the particle; $Z$ and $A$ are the atomic number and mass, respectively; $K=4 \pi N_{A} e_{e}^{2} m_{e} c^{2} ; m_{e}$ is the electron mass; $r_{e}$ is the classical radius of the electron; $N_{A}$ is Avogadro's number; and, $I$ is the mean excitation energy. The energy loss of various particles in various materials, calculated using the Bethe formula, is shown in Figure 6.

In proton-proton collisions as the LHC electrically charged particles can only be highly ionizing if they are massive and thus moving very slowly with $\beta \gamma<1$, and/or multiply charged. However, there are no known massive stable or long-lived particles that are multiply charged. The only way that a Standard Model particle can be highly ionizing is if it is slowing down to stop. Thus, massive stable or pseudo-stable HIPs traversing the detector are clear messengers of new physics with no obvious Standard Model Backgrounds. The Bethe formula for ionization energy loss by a relativstic monopoles is given by replacing $z e$ with $n g \beta$ :

$$
-\frac{d E}{d x}=(n g / e)^{2} \frac{K Z}{A}\left[\frac{1}{2} \ln \left(\frac{2 m_{e} \beta^{2} \gamma^{2}}{I^{2}}\right)-\beta^{2}\right]
$$

Dirac showed the magnetic charge of a monopole is $g=n(e / 2 \alpha)$, where $\alpha(\approx 1 / 137)$ is the fine structure constant. Thus, a unit of magnetic monopole is $g \sim 68.5 \mathrm{e}$. As we can see from the Bethe formula for magnetic charge given above, the energy loss of a singly charged $(n=1)$ very relativistic magnetic monopole $(\beta \approx 1)$ is amazingly about 4700 times that of a proton!

MoEDAL's sensitivity to highly ionizing messengers of new physics, together with its ability to make a permanent "photographi'c' record of HIP BSM particles in its NTD detectors and capture such particles in its trapping detectors, is unique. MoEDAL's physics aims - developed in two workshops held at CERN in June 2013 and 2014 - are to use HIPs to explore the discovery horizon at the LHC as described in the MoEDAL collaboration's recent paper, "The Physics Programme of the MoEDAL Experiment at the LHC" [2]. The various scenarios accessible to MoEDAL are listed here:

Scenarios involving magnetically charged particles

- Monopole-like structures in the electroweak theory

- Electroweak Monopole

- The Cho-Maison Mass Estimate

- Singularity resolution within string/brane theory

- Electroweak strings

- Vacuum decay and light 't Hooft-Polyakov monopoles

- Monopolium 
Electrically-Charged Massive (Meta-) Stable Particles in Supersymmetric Scenarios

- Metastable lepton NLSP in the CMSSM with a neutralino LSP

- Metastable sleptons in gravitino LSP scenarios

- Metastable stop squark scenarios

- Long-lived gluinos in split supersymmetry

- Supersymmetric scenarios with R -parity violation

- Heavy sleptons from Gauge Mediated Supersymmetry Breaking scenarios

- Metastable charginos

- The Fat Higgs model

- XYons from 5D SUSY breaking

Scenarios with Extra Dimensions

- Extra dimensions and microscopic black holes

- Long-lived microscopic black holes

- Microscopic black hole remnants

- Long-lived Kaluza-Klein particles from Universal Extra Dimensions

- D-particles with magnetic charge

- Electrically-charged D-particles

Highly-Ionizing Particles in Other Scenarios

- Long-lived heavy quarks

- Massive (pseudo-) stable particles from vector-like confinement

- Fourth-generation fermions

- 'Terafermions' from a 'sinister' extension of the Standard Model

- A massive particle from a simple extension to the SM

- Fractionally charged massive particles

Scenarios with Doubly-Charged Massive Stable Particles

- XY gauginos and warped extra dimension models

- Doubly-charged leptons in the framework of walking technicolor models

- Doubly-charged Higgs bosons in the L-R symmetric model

- Doubly Charged Higgsinos in the L-R supersymmetric model

- Doubly-charged leptons in the framework of almost commutative geometry

Highly-Ionizing Multi-Particle Excitations

- Q-balls

- Strangelets

- Quirks 


\section{Conclusion}

The Standard Model paradigm still leaves a great many unanswered fundamental questions at the Terascale and beyond. Thus, the emphasis must now fall on the search for new physics that heralds a deeper more fundamental theory, surpassing the SM. The LHC has risen to this challenge as is admirably demonstrated by the 350 "search" papers published by ATLAS and CMS - roughly half their total paper output, to date [17].

The singular and seminal concept behind MoEDAL's new light on the high-energy frontier is the use of massive HIPs, for which there are no SM counterparts, as direct probes of visionary new physics at the Terascale. On the other hand the main LHC detectors, that currently represent the state of the art, mostly infer the presence of physics BSM by relying on detecting SM products of such new physics - for which there are significant conventional backgrounds.

MoEDAL is a high-risk but revolutionary gain experiment that builds on close interactions between theoretically very well motivated "blue-sky" research and mainstream strategic research at the Terascale horizon. The "high risk" aspect of MoEDAL's broad discovery physics program is validated by the possibility of a revolutionary breakthrough with major impact beyond the field of particle physics. The audacious nature of MoEDAL's quest is balanced by the guarantee of an extensive search program that can place unparalleled bounds on BSM in areas inaccessible, or barely accessible, to the main LHC experiments, expanding the new physics reach of the conventional LHC experiments

\section{References}

[1] J. L. Pinfold, CERN-LHCC-2009-006 ; MoEDAL-TDR-001 (2009).

[2] B. Acharya et al., "The Physics Program of the MoEDAL Experiment at the LHC", MoEDAL Collaboration, Int. J. Mod. Phys. A29, No. 231430050 (91 pages), (2014).

[3] http://aladdin.utef.cvut.cz/ofat/others/Timepix/

[4] A. de Roeck et al.,"Sensitivity of LHC Experiments to Exotic Highly Ionising Particle", Eur. Phys. J. C72, (2012).

[5] K. Bendtz et al., "Search in $8 \mathrm{TeV}$ proton-proton Collisions with the MoEDAL Monopole Trapping Test Array”, e-Print:arXiv:1311.6940(physics.ins-det], (2013)

[6] Beringer et al. (Particle Data Group), PR D86, 0100012012 and 2013 update for the 2014 edition; https://pdg.web.cern.ch/pdg/2012/reviews/rpp2012-rev-mag-monopole-searches.pdf.

[7] G.R. Kalbfleisch et al., "Limits on Production of Magnetic Monopoles Utilizing Samples from the D0 and CDF Detectors at the Tevatron", Phys.Rev.D69, 052002 (2004); K. Bendtz, et al., "Search for Magnetic Monopoles in Polar Volcanic Rocks", Phys. Rev. Lett. 110, 121803 (2013).

[8] P. A. M. Dirac, "Quantised Singularities in the Electromagnetic Field". Proc. Roy. Soc. Lond. A 133, 60 (1931);

[9] G. 't Hooft, "Magnetic Monopoles in Unified Gauge Theories", Nucl. Phys. B 79, 276 (1974);

[10] A. M. Polyakov, "Particle Spectrum in the Quantum Field Theory", JETP Lett. 20, 194 (1974);

[11] H. Georgi and S.L. Glashow, “ Unified weak and electromagnetic interactions without neutral currents ", Phys. Rev .Lett. 28, 1494 (1972).

[12] J. Callan and G. Curtis, "Monopole Catalysis of baryon Decay", Nucl.Phys. B212, 391 (1983).

[13] J. Preskill, “Magnetic Monopoles”, Ann. Rev. Nucl. Part. Sci. 34, pp461-530 (1984).

[14] Y.M. Cho, D. Maison, "Monopoles in Weinberg-Salam Model”, Phys. Lett. B391, 360 (1997).

[15] Y. M. Cho, K. Kim and J. H. Yoon, "Mass of the Electroweak Monopole", e-Print: arXiv:1212.3885[hep-ph] (2012). 
[16] H. Bethe und J. Ashkin, "Experimental Nuclear Physics", J. Wiley, New York, 253 (1953); Particle Data Group, Eur. Phys. J. C3, 144 (1998).

[17] This result is derived from the INSPIRE database that contains a full record of ATLAS and CSM Publications. 